\title{
Reconstruction of the Medical Collateral Ligament with Degradable, Elastic Internal Bracing
}

\author{
Daniel L Rodkey, MD ${ }^{1 *}$, Boddy G Yow, MD $D^{1}$ and Brandon J Bryant, $M D^{2}$ \\ ${ }^{1}$ Walter Reed National Military Medical Center Bethesda, USA \\ ${ }^{2}$ Inova Fairfax Medical Center Falls Church, USA
}

\begin{abstract}
The medical collateral ligament $(\mathrm{MCL})$ is the most commonly injured knee ligament and has a large capacity for healing. The majority of isolated $\mathrm{MCL}$ injuries may be treated conservatively due to its capacity to heal. However, acute $\mathrm{MCL}$ disruptions associated with multiligamentous knee injuries, distal avulsions and chronic MCL attenuation may benefit from surgical intervention to prevent valgus instability. Several treatment strategies have been proposed to include primary repair, primary repair with autograft/allograft/internal bracing augmentation and reconstruction with autograft/allograft/internal bracing. In this surgical technique article, we present the technique of acute $\mathrm{MCL}$ repair with degradable, flexible internal bracing. With this technique, early rehabilitation is safe because of the internal bracing. Unlike permanent, rigid internal bracing, degradable, flexible internal bracing prevent stress shielding of the ligament while providing support throughout the healing cascade. Advantages therefore include earlier rehabilitation, faster recovery, and physiologic stress to ligaments after healing completion.
\end{abstract}

\section{Introduction}

Healing of ligamentous injuries is dependent on ligament location, intrinsic factors surgical factors and rehabilitation [1]. The extraarticular nature and ability to adequately brace the MCL often make it amenable to nonoperative treatment. It the setting of concomitant ACL tear, there is evidence of improved motion for those who are treated nonoperatively [2]. However, in situations with multiligamentous knee injuries, interstitial Grade III tear, surgical intervention may be warranted.

The motivation for MCL repair or reconstruction is to protect other ligamentous repairs by increasing physiologic constraint to the knee. Additionally, repairing Grade II or Grade III tears helps to prevent late valgus instability. Achieving the proper amount of support and stress on a healing ligament is paramount. The healing process consists of hemorrhage, inflammation, repair and remodeling [1]. Animal models have shown the ligament is weakest during the inflammatory period which occurs about 6-8 weeks after injury or reconstruction $[3,4]$. With this knowledge some authors have advocated for internal bracing of the knee to protect attenuation or failure during the healing process [5]. This bracing technique is rigid and permanent. In this article we describe the surgical technique of $\mathrm{MCL}$ reconstruction with a degradable, flexible internal brace. This presented bracing technique may be useful in isolated or combined injuries as well as repair or recon- struction procedures.

\section{Surgical Technique}

In this article, we describe the reconstruction of a complete MCL tear utilizing Flexband (Artelon Marietta, GA) which is a poly (urethane urea) material. In should be noted that despite this case being performed in a patient with multiligamentous knee injury to include anterior cruciate ligament, posterior cruciate ligament and medial collateral ligament, this same technique can be performed for isolated $M C L$ tears. The MRI demonstrates a complete tear of the MCL (Figure 1a).

\section{Patient positioning}

The patient is placed in the supine position, and the oper-

\footnotetext{
*Corresponding author: Daniel L Rodkey, MD, Walter Reed National Military Medical Center Bethesda 8901 Wisconsin Ave, Bethesda, MD 20889, USA, Tel: 508-479-2849

Accepted: May 04, 2021

Published online: May 06, 2021

Citation: Rodkey DL, Yow BG, Bryant BJ (2021) Reconstruction of the Medical Collateral Ligament with Degradable, Elastic Internal Bracing. J Orthop Surg Tech 4(1):262-265
} 

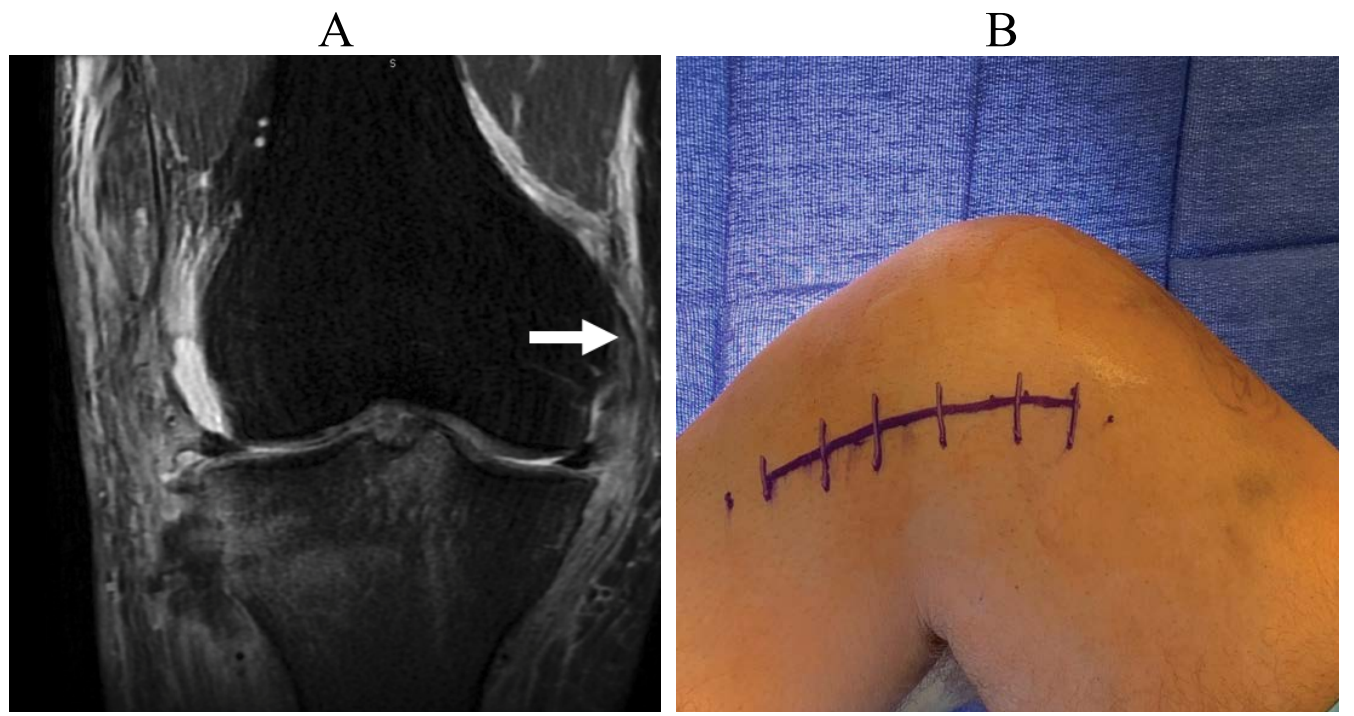

Figure 1: A) Coronal T2-weighted MRI image of the right knee showing midsubstance medical collateral ligament tear. The arrow demonstrates the superficial MCL stump still attached to femoral origin; B) View of the medial side of right knee demonstrating longitudinal skin incision between medial epicondyle and $\mathrm{MCL}$ insertion.

ative leg is prepared and draped for standard knee arthroscopy. The foot of the bed remains extended while addressing MCL pathology. A foot post is placed to achieve approximately 30 degrees of knee flexion.

\section{Allograft and augment preparation}

An augmented semitendinosus allograft tendon was prepared. Both ends of the autograft were whip stitched and placed into a standard graft holding device. The graft was then tensioned to approximately $20 \mathrm{Nm}$ for 15 minutes. Following tensioning, a $0.7 \times 16 \mathrm{~cm}$ Artelon Flexband augment was positioned in the center of the allograft. The Flexband is symmetric, in that either side may interface with the tendon. The Flexband is then whipstitched to the allograft at the ends. Typically, 5 throws is sufficient. Excess allograft longer than the augment is trimmed. The augmented tendon graft was sized to $6 \mathrm{~mm}$.

\section{$M C L$ reconstruction}

A skin incision is made at the medial epicondyle and extended distally to the anatomic insertion of the MCL (Figure 1b). Attention is directed first towards the medial epicondyle to identify the proximal $\mathrm{MCL}$ footprint and residual stump. Dissection is continued through layer 1 of the knee to the sartorial investing fascia. The fascia was incised superior and proximal to the sartorius exposing the sub-pes bursa with superficial MCL. If no residual stump is identifiable, or the tissues are too traumatized, fluoroscopy will help confirm location of superficial MCL. The footprint, located just posterior and proximal to the medial epicondyle [6]. After identifying the $S M C L$ foot print, evaluate surrounding tissues for damage and repair at this time. At the MCL footprint, pass a Beath pin.

The distal tibial insertion is addressed next. The skin incision is developed to expose sartorius fascia, which is incised. The pes tendons are retracted distally to expose the $\mathrm{MCL}$ insertion at approximately $6 \mathrm{~cm}$ from the joint line and $1 \mathrm{~cm}$ medial to the tibial tuberosity [1]. The anchor location is marked with a Beath pin. Using the two pins, evaluate isometry through a range of motion. Isometric position is important to a functional MCL through physiologic range of motion.

After confirming isometry, drill a $7 \mathrm{~mm}$ socket to a depth of $30 \mathrm{~mm}$ at the $\mathrm{MCL}$ insertion site on the femur. Advance the Beath pin out of the distal lateral thigh and maintain suture from Beath pin for passing of the graft. Next drill a $7 \mathrm{~mm}$ socket to a depth of $20 \mathrm{~mm}$ at the MCL insertion site on the tibia. Tap with both sockets with a $7 \mathrm{~mm}$ tap. The graft is inserted first into the tibial socket and secured using a $7 \times 23 \mathrm{~mm} \mathrm{Ar}$ threx Bio Composite tenodesis screw (Figure $2 \mathrm{a}$ and Figure $2 b)$ Ensure that the Artelon graft augment is superficial to the allograft and the joint for biomechanical advantage [7]. The free, proximal portion of the graft is passed into the MCL origin site using the passing suture. With the knee is positioned in 20 degrees of flexion, a $7 \times 25 \mathrm{~mm}$ Regenesorb interference screw is placed. Next a $1.8 \mathrm{~mm}$ Q-Fix suture anchor is placed 12 to $15 \mathrm{~mm}$ distal to the joint line at the proximal tibial flare. The free suture ends of the anchor are passed through the tendon and Artelon structure in a mattress fashion to secure the $\mathrm{MCL}$ at the proximal of the two tibial insertions. Prior to closing, inspect again for any unaddressed medial sided injuries. Ensure that patient is able to achieve full extension and is stable to valgus resistance at 0 and 30 degrees.

\section{Rehabilitation protocol}

The presence of concomitant injury will influence rehabilitation protocol. The primary objective is to encourage early motion in order to prevent stiffness and arthrofibrosis. These goals of early motion must be weighed against protecting the repair. A hinged knee brace is applied post-operatively and the patient is permitted to begin range of motion post-operative day 1 . Weight bearing is allowed while locked in extension until adequate return of quadriceps function. The brace is typically worn for 4 weeks, after which it may be weaned and standard MCL rehabilitation program is followed. 
A

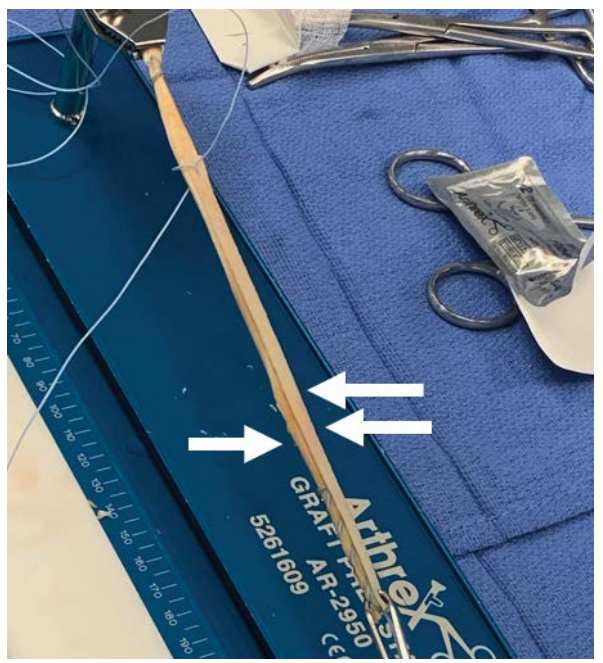

$\mathrm{B}$

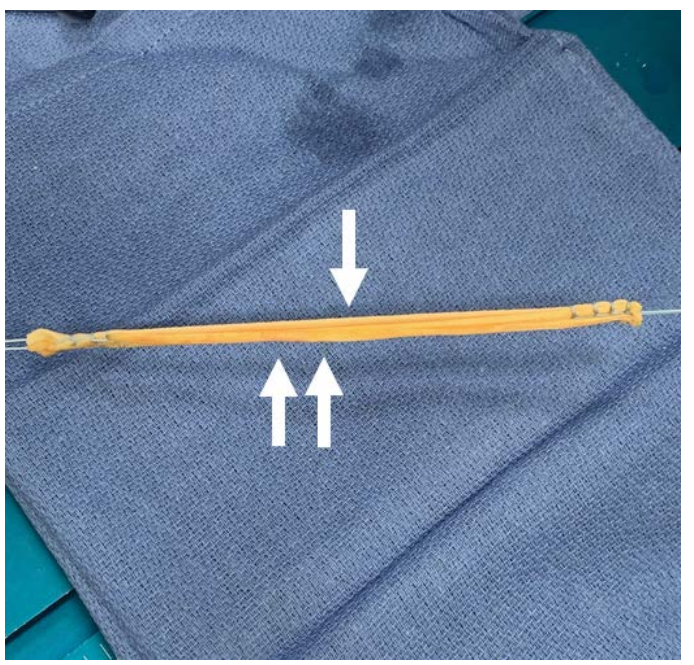

Figure 2: A) Graft holding device containing Achilles allograft (single arrow) and Artelon augment (double arrow). The augment is attached to the allograft through standard whipstitch technique; B) The augmented allograft is tensioned and trimmed to the proper size.

A

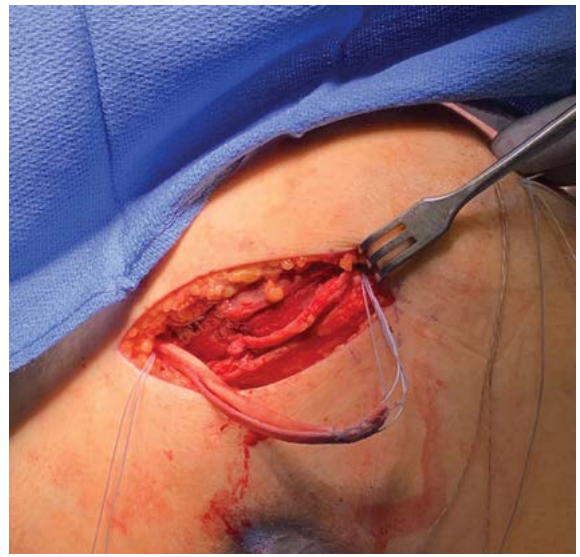

B



Figure 3: A) Augmented allograft is fixed at the tibial side with interpherence screw; B) Augmented allograft is brought to MCL origin to confirm isometry prior to final fixation.

\section{Discussion}

The treatment of MCL tear with degradable, flexible augmentation is an incremental improvement in the evolution of $\mathrm{MCL}$ reconstruction; Artelon is one such example of degradable, flexible internal bracing. We describe this technique of $\mathrm{MCL}$ reconstruction with degradable, flexible augmentation as it affords several theoretical benefits. The two biggest advantages over permanent, rigid bracing is that the implant is temporary and the implant allows the repaired or reconstructed graft to undergo appropriate stress following the healing process.

After indicating a patient for surgical fixation of the $M C L$, there are multiple ways to fix the injury. Generally, if the injury is amenable to direct repair, this is the preferred treatment option [8]. The repair may be augmented with autograft, allograft or internal bracing. In patient with chronic medial knee injuries or serve, unrepairable midsubstance injuries, reconstruction is generally recommended [8]. The reconstruction may be augmented with internal bracing. Internal bracing may be categorized as permanent, rigid or degradable, flexible (Figure $3 a$ and Figure $3 b$ ).

The concept of internal bracing was described by Lubowitz for anatomic repair of medical knee injuries [9]. Internal bracing aims to protect the ligament through the healing process, mitigate risks of patient noncompliance, minimize secondary injury or attenuation during rehabilitation. However, the internal brace described by Lubowitz is composed of ultrahigh-molecular weight polyethylene/polyester (Fiber Tape; Arthrex). This material is stiffer than more elastic native 
A

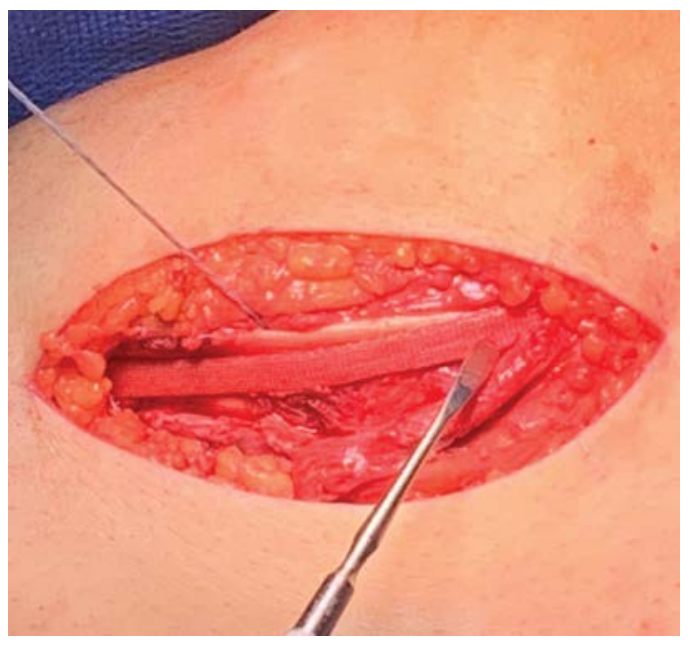

B

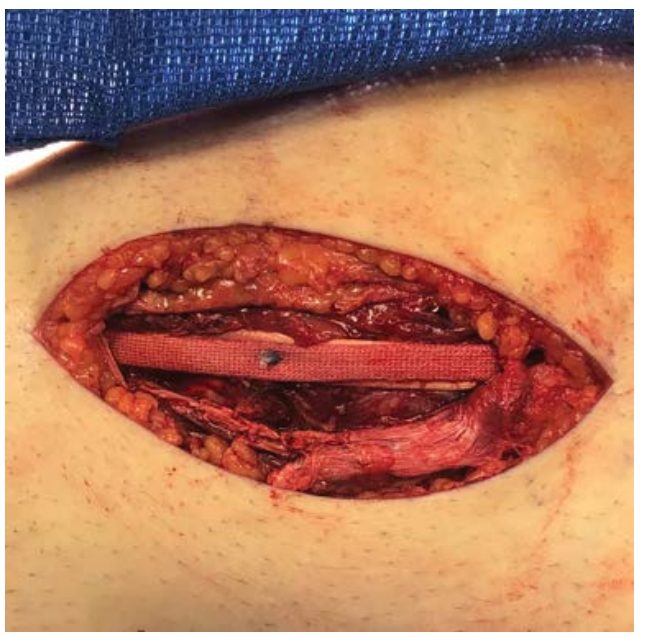

Figure 4: A) The Artelon augment is place on the superficial side of the tendon graft for maximum biomechanical advantage; B) Augmented allograft is fixed at the tibial side with interpherence screw; B) Final fixation of augmented allograft demonstrating proximal tibial insertion of $\mathrm{MCL}$ fixed with suture anchor.

tendons. This may predispose the patient to stress shielding of healed ligaments. Polyethylene/polyester does not degrade. In contrast, the Flexband poly (urethane urea) material better approximates the stiffness of tendon and begins degrading at 24 months $[10,11]$.

In conclusion, we have presented the surgical technique of $\mathrm{MCL}$ reconstruction with internal bracing using degradable, flexible Artelon augmentation (Figure $4 a$ and Figure 4b). To date, the utilization of Artelon augmentation has not been described for $\mathrm{MCL}$ repair or reconstruction. With this technique, medial sided structures may be repaired or reconstructed, and the internal bracing with Artelon allows for early range of motion, valgus stability, prevention of secondary injury and early rehabilitation while minimizing the risks of over-constraint of medial structures, stress shielding of healed ligaments, or permanent internal brace.

\section{References}

1. Woo SL, Vogrin TM, Abramowitch SD (2000) Healing and repair of ligament injuries in the knee. J Am Acad Orthop Surg 8: 364372.

2. Millett PJ, Pennock AT, Sterett WI, et al. (2004) Early ACL reconstruction in combined ACL-MCL injuries. J Knee Surg 17: 94-98.

3. Butler DL, Grood ES, Noyes FR, et al. (1989) Mechanical properties of primate vascularized vs. nonvascularized patellar tendon grafts; changes over time. J Orthop Res 7: 68-79.
4. Manske RC, Prohaska D, Lucas B. (2012) Recent advances following anterior cruciate ligament reconstruction: Rehabilitation perspectives : Critical reviews in rehabilitation medicine. Curr Rev Musculoskelet Med 5: 59-71.

5. Van der List JP, Di Felice GS (2017) Primary repair of the medial collateral ligament with internal bracing. Arthrosc Tech 6: e933-e937.

6. La Prade MD, Kennedy MI, Wijdicks CA, et al. (2015) Anatomy and biomechanics of the medial side of the knee and their surgical implications. Sports Med Arthrosc Rev 23: 63-70.

7. Peterson L, Eklund U, Engstrom B, et al. (2014) Long-term results of a randomized study on anterior cruciate ligament reconstruction with or without a synthetic degradable augmentation device to support the autograft. Knee Surg Sports Traumatol Arthrosc 22: 2109-2120.

8. Laprade RF, Wijdicks CA (2012) The management of injuries to the medial side of the knee. J Orthop Sports Phys Ther 42: 221233.

9. Lubowitz JH, MacKay G, Gilmer B. (2014) Knee medial collateral ligament and posteromedial corner anatomic repair with internal bracing. Arthrosc Tech 3: e505-508.

10. Liljensten E, Gisselfalt K, Edberg B, et al. (2002) Studies of polyurethane urea bands for $A C L$ reconstruction. J Mater Sci Mater Med 13: 351-359.

11. Artelon tissue reinforcement implants tensile testing: Technical Report 061.

DOI: $10.36959 / 453 / 558$

Copyright: (c) 2021 Rodkey DL, et al. This is an open-access article distributed under the terms of the Creative Commons Attribution License, which permits unrestricted use, distribution, and reproduction in any medium, provided the original author and source are credited. 